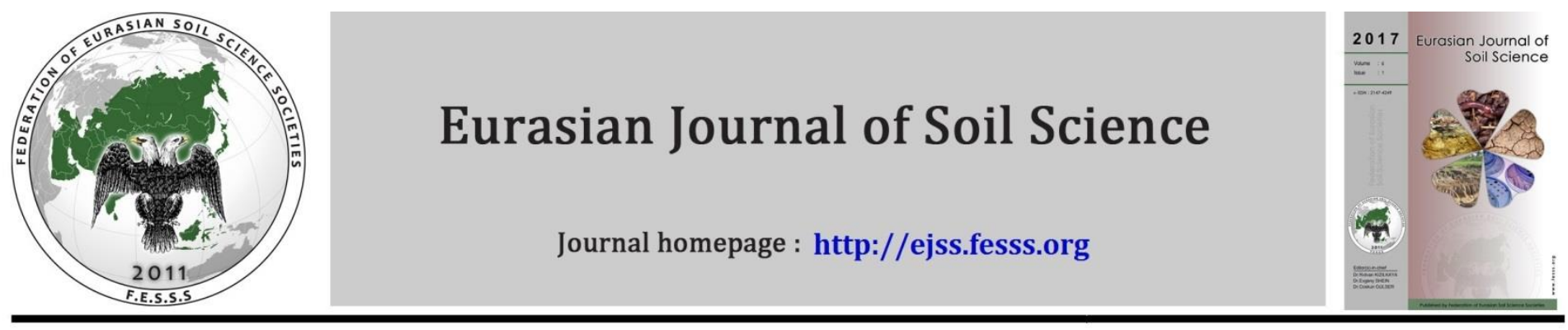

\title{
Conductive and steam-diffuse constituents of thermotransfer in different soil moisture contents: case study of the Altai Region's soils
}

\author{
Sergey V. Makarychev, Andrey G. Bolotov*
}

Altai State Agricultural University, Barnaul, Russia

\begin{abstract}
The goal of this study was to determine the conductive and steam-diffusive heat transfer constituents in the soil. Based on the solution of differential equation system of heat and mass transfer, the method to determine the conductive and steam-diffusive heat transfer constituents in wet soils was developed. To measure the thermophysical properties in laboratory setting, a pulse method of a two-dimensional heat source was used. The method takes into account the patterns of temperature field equalization in an unbounded medium after the heat source termination. A feature of this process is the occurrence of peak temperature at the investigated point of the medium at a given instant. In this experiment, the temperature was controlled not only at the investigated point of the medium, but also at the soil-heater interface. The proposed method was used to study the thermophysical indices of the chernozems of the Altai Region's Priobye area (the Ob River area). The chernozem under study is of light-loamy particlesize composition; the illuvial horizon $B$ is enriched by a sand fraction. It has been found that the soil conductive thermal diffusivity is reduced with increasing moisture content. The steam-diffusive thermal diffusivity has the extremum at the moisture close to the discontinuous capillary moisture. In humus horizons it plays a smaller role than in the mineral horizons. The thermal diffusivity determined by the steam molecule motion in the pore space of the soil exceeds the conductive thermal diffusivity two or three times. At the same time thermal steam diffusivity is more than ten time lower than the conductive constituent. Eventually, the stem molecules though dramatically accelerating the processes of heat transfer in the soil profile conduct a small amount of heat and make a weak contribution to soil thermal accumulation.
\end{abstract}

Accepted : 09.06.2016

Keywords: Soil, thermal capacity, thermal conductivity, thermal diffusivity, thermotransference in soils, conductive and steam-diffuse constituents.

(C) 2017 Federation of Eurasian Soil Science Societies. All rights reserved

\section{Introduction}

The processes of thermal accumulation and thermal transference in soil profile are a great deal determined by heat capacity, thermo and thermometric conductivity of soil. These qualities depend on mechanical composition, organic matter content, moisture content, density and other soil-physical factors (Nerpin and Chudnovskiy, 1970; Gülser and Ekberli, 2004; Mikayilov and Shein, 2010; Arkhangelskaya, 2014). This predetermines big practical possibilities for directed coercion on the soil climate, and therefore, on the processes and life conditions of plants.

\footnotetext{
${ }^{*}$ Corresponding author.

Altai State Agricultural University, Barnaul, Altai Krai 656031 Russia

Tel.: + 73852628353

e-ISSN: $2147-4249$
} 
The chernozem of the Priobye area, formed in the Altai Region, is the most promising in terms of water-andheating meliorations, causing the raise in productivity. The efficiency of the used agronomic practices should be scientifically substantiated which demands a detailed research of thermo-physical parameters of chernozem.

The heat transfer in moist soil was studied by a number of researchers (Globus, 1983; Globus, 1987; Lunin, 1972; Panfilov and Makarychev, 1981; Philip and De Vries, 1957); their works developed the concept of soil moisture heat transfer. The concept is based on the acknowledgement of the mixed heat-and-moisture mechanism of thermo-moisture transference, which includes conductive thermo-transference through firm and liquid phases and diffuse transference of heat by the streams of steam and liquid moisture. The mixed mechanism nowadays is the most acknowledged in soil thermo-physics. Nevertheless, the development of the theory of the mixed mechanism of thermo-moisture exchange made us introduce, for concordance of theory and experiment, the so-called reinforcement factors (Globus, 1983), based on the row of assumptions (Globus, 1987), not always objectively reflecting real conditions formed in soil.

This leads to some mistakes by the determination of heat-stream components caused by thermo-diffusion of steam and liquid moisture, and also its phase transformations, especially when the moisture content is less or more than the critical one (Globus, 1983). The goal of this study was to determine the conductive and steam-diffusive heat transfer constituents in the soil.

\section{Material and Methods}

According to the research goal, the leached chernozem of light-loamy particle-size composition of the Priobskoye Plateau of the Altai Region and quartz sand were studied (Makarychev and Mazirov, 1996; Bolotov and Makarychev, 2015). The samples of undisturbed structures in different soil horizons were studied.

To measure the thermophysical properties in laboratory setting, a pulse method of a two-dimensional heat source was used. The method takes into account the patterns of temperature field equalization in an unbounded medium after the heat source termination. A feature of this process is the occurrence of peak temperature at the investigated point of the medium at a given instant (Chudnovskiy, 1976). The knowledge of this temperature and time enables to determine the soil thermal capacity, thermal conductivity and thermal diffusivity.

\section{Results and Discussion}

We made an attempt to find directly the conductive and steam-diffuse constituents of thermotransference on the base of using impulse method of flat heater, when the stream of liquid moisture does not influence the results of the experiment (Lunin, 1972; Tikhonov and Arsenin, 1979). The suggested method is based on the solution of the so-called inverse problem (Anikonov and Bubnov, 1981; Lavrentyev, 1986). We solved the system of differential equations of heat-and-mass transference (Lunin, 1972) for it.

$$
\begin{array}{r}
C_{h} \gamma_{h} \frac{\partial t_{h}}{\partial \tau}=\lambda_{h} \frac{\partial^{2} t_{h}}{\partial x^{2}}+W \\
C \gamma \frac{\partial t}{\partial \tau}=\lambda \frac{\partial^{2} t}{\partial x^{2}}+r \cdot I_{s} \\
C_{s} \gamma_{s} \frac{\partial P}{\partial \tau}=\mu \frac{\partial^{2} P}{\partial x^{2}}
\end{array}
$$

with initiate $t(0, x)=t_{0}, x \geq R$ and limited conditions $t(\tau, R)=\varphi(\tau), \tau \geq 0$.

If the temperature on the borderline of the heater and the investigated pattern is measured in the process of experiment, there is no need then to solve the equation (1.1), which characterizes the parameters of the heater.

In this case it is sufficient to consider the following problem: 


$$
\begin{array}{r}
C \gamma \frac{\partial t}{\partial \tau}=\lambda \frac{\partial^{2} t}{\partial x^{2}}+r \cdot I_{s} \\
C_{s} \gamma_{s} \frac{\partial P}{\partial \tau}=\mu \frac{\partial^{2} P}{\partial x^{2}} \\
t(0, x)=t_{0}, x \geq R \\
t(\tau, R)=\varphi(\tau), \tau \geq 0
\end{array}
$$

Note: $\quad c$ - specific heat of soil, $\gamma$ - soil density, $C$ - specific heat of steam, $\gamma_{s}$ - steam density, $\gamma_{s}=M P / R T$, $\gamma / P=K, \lambda$ - conductive thermal conduction, $\mu$ - coefficient of a team permeability, $r$ - specific heat of vaporization, $t$ - soil temperature, $P$ - pressure of saturated steam in porous space of soil, $\tau$ - time, $x$ - distance from the heater, $I_{s}$ - capacity of conditional heat source caused by steam transference.

As the initial condition (2.3) and limited condition (2.4) are given, then it's defind:

$$
P(t(0, x))=P\left(t_{0}\right)=P_{0}
$$

and

$$
P(t(\tau, R))=\psi(\tau)
$$

If we look at the equation (2.2) with the conditions (2.5), (2.6), we shall see, that the solution $P(\tau)$ of this mixed problem exists and it is sole.

Substituting the received $\frac{\partial P}{\partial \tau}$ in $(2.1)$ we shall come to equation:

$$
C \gamma \frac{\partial t}{\partial \tau}=\lambda \frac{\partial^{2} t}{\partial x^{2}}+k r \frac{\partial P}{\partial \tau}
$$

with initial

$$
t(0, x)=t_{0}, x \geq R
$$

and limited conditions:

$$
t(\tau, R)=\varphi(\tau), \tau \geq R
$$

Hence it appears that the solution of the system (2) exists and it is sole, because it is correct.

It is necessary to define coefficient $\mu$ in the equation (2.2) for direct solution of the system. It will demand additional data, i.e. the measuring of the temperature in the moment of time $\tau^{*}$ in point $x^{*}+R, \tau^{*}>0, x^{*}>0$ and $t\left(\tau^{*}, x^{*}+R\right)$.

Taking it all into consideration, we shall get the problem, including the equation (2.2), conditions (2.5), (2.6) and the condition $P\left(t\left(\tau^{*}, x^{*}+R\right)\right)=\wp\left(\tau^{*}\right)$.

defining $P(t(\tau, x+R))=U(\tau, x), a^{*}=\frac{\mu}{C_{s} \gamma_{s}}$

we shall have:

$$
\frac{\partial U}{\partial \tau}=a^{*} \frac{\partial^{2} U}{\partial x^{2}}
$$




$$
\begin{array}{r}
U(0, x)=\varphi(0) \\
U(\tau, x)=\psi(\tau), \tau \geq 0 \\
U\left(\tau^{*}, x^{*}\right)=\wp\left(\tau^{*}\right)
\end{array}
$$

To find $a^{*}$, i.e. to solve the inversed problem, it is necessary to know the temperature on the heater surface $t(\tau, R)$ and on the distance $x^{*}$ from it $t\left(\tau^{*}, x^{*}+R\right)$ at the moment of time $\tau^{*}$.

So, $\varphi(0)=P\left(t_{0}\right)$

$\psi(\tau)=P(t(\tau, R))$

$\wp\left(\tau^{*}\right)=P\left(t\left(\tau^{*}, x^{*}+R\right)\right)$ will be given.

Finally, expressing it from equation (2.2) $\mathfrak{J}_{s}=\mu \frac{\partial^{2} P}{\partial x^{2}}$ and substituting the given dependence in (2.1) we shall get:

$C \gamma \frac{\partial t}{\partial \tau}=\lambda \frac{\partial^{2} t}{\partial x^{2}}+k r \mu \frac{\partial^{2} P}{\partial x^{2}}$

Calculating:

$$
\frac{\partial P}{\partial x}=\frac{\partial}{\partial x}(P(t(\tau, x)))=\frac{\partial P}{\partial \tau} \cdot \frac{\partial t(\tau, x)}{\partial x}
$$

we shall find: $\quad \frac{\partial t}{\partial \tau}=\frac{\partial}{\partial x}\left(\left(\frac{\lambda+\frac{k r \mu}{C_{s} \gamma_{s}} \cdot \frac{\partial P}{\partial \tau}}{C \gamma}\right) \frac{\partial t}{\partial x}\right)$

This is a well-known equation of thermoconductivity-quasi-linear, parabolic; and the coefficient.

$$
\lambda+\frac{k r \mu}{C_{s} \gamma_{s}} \cdot \frac{\partial P}{\partial t}
$$

is the, total thermal conduction of soil, and

$$
\frac{k r \mu}{C_{s} \gamma_{s}} \cdot \frac{\partial P}{\partial t}
$$

is the constituent of thermal conduction, caused by steam-transference.

The direct calculation of thermometric conductivity $a^{*}$, caused by phase transformation of moisture on the "warm" and "cold" sides of water cuffs and the movement of steam molecules in the porous space is done with the help of computer program, and the temperature values on the surface of the heater and in the studied point of damp soil at definite moments were used as initiate data.

The values of partial pressure of steam, necessary for calculation, were defined by the known table (Kay and Laby, 1995).

The suggested method of getting steam-diffuse parameters of thermotransference was used while making an experimental research in leached chernozem with light-loamy mechanical structure of the Priobskoye Platean on the Altai Region, and for comparison, of quartz sand. According to the data of the Department of Soil Science of the Altai Agricultural Institute, the chernozem under study is of light-loamy particle-size composition (Table 1); illuvial horizon $B$ is enriched by sand fraction.

The volume weight of the upper humus-accumulating layers varies from 1200 to $1160 \mathrm{~kg} / \mathrm{m}^{3}$, rising in the 
horizon $B$ to $1330 \mathrm{~kg} / \mathrm{m}^{3}$. Correspondingly, the total porosity is lower there, just as the porosity of aeration by the field moisture capacity $(F C)$ is the lowest in the arable layer.

The minimum moisture-capacity of genetic horizons of chernozem decreases with the depth. The capillary bond breaking moisture $(C B B)$ changes analogically.

Down the profile the number of organic matter decreases very much (from 5.2 per cent to 0.5 per cent correspondingly in the horizons $A$ and $B$ ).

Table 1. Physical-mechanical and hydro-physical characteristics of leached chernozem

\begin{tabular}{|c|c|c|c|c|c|c|c|}
\hline \multirow{3}{*}{ Soil Horizon } & \multicolumn{2}{|c|}{ Porosity } & \multicolumn{2}{|c|}{ Fractions } & \multicolumn{3}{|c|}{ Hydroconstants } \\
\hline & Total & Aeration & Clay & Sand & $W P$ & $C B B$ & $F C$ \\
\hline & \multicolumn{4}{|c|}{$\%$} & \multicolumn{3}{|c|}{$\%$ from weight } \\
\hline$A$ & 52.2 & 15.6 & 29.0 & 34.9 & 6.9 & 23.1 & 30.3 \\
\hline$A B$ & 54.0 & 22.5 & 26.5 & 29.5 & 6.9 & 20.6 & 27.2 \\
\hline$B$ & 48.4 & 20.7 & 26.2 & 52.3 & 7.0 & 17.0 & 20.8 \\
\hline
\end{tabular}

WP - wilting point

The investigated quartz sand with predominating $2 \mathrm{~mm}$ faction has volume weight $1580 \mathrm{~kg} / \mathrm{m}^{3}$, total porosity 40.3 percent. Because of field moisture capacity (only 6 percent of total weight) the porosity of aeration reaches 53 percent of volume of porous space. Some results of thermophysical research are represented in Table 2. The data of Table 2 shows that the conductive thermometric conductivity of genetic horizons of leached chernozem and quartz sand has the tendency to decrease with the rise of moisture content. One can see it especially in the sand and in the illuvial horizon $B$, containing a little amount of humus. Conductive thermometric conductivity in them lowers from $0.4 \times 10^{-6}$ to $0.16 \times 10^{-6} \mathrm{~m}^{2} / \mathrm{s}$ and from $0.36 \times 10^{-6}$ to $0.17 \times 10^{-6} \mathrm{~m}^{2} / \mathrm{s}$, respectively.

Table 2. Conductive (numerator) and steam-diffuse (denominator) thermometric conductivity of chernozem and quartz sand by different degrees of moistening $\left(a ; a^{*} \times 10^{-6} \mathrm{~m}^{2} / \mathrm{s}\right)$

\begin{tabular}{|c|c|c|c|c|}
\hline \multicolumn{5}{|c|}{ Leached chernozem } \\
\hline Soil Horizon & Absolutely dry & & & \\
\hline \multirow[b]{2}{*}{ A } & \multirow[b]{2}{*}{0.36} & 0,35 & 0,33 & 0,30 \\
\hline & & 0,13 & 0,16 & 0,13 \\
\hline \multirow[b]{2}{*}{$\mathrm{AB}$} & \multirow{2}{*}{0.40} & 0,33 & 0,32 & 0,32 \\
\hline & & 0,19 & 0,29 & 0,24 \\
\hline \multirow{2}{*}{ B } & \multirow{2}{*}{0.36} & 0,16 & 0,17 & 0,16 \\
\hline & & 0,33 & 0,39 & 0,36 \\
\hline & & Quart & & \\
\hline \multirow[t]{3}{*}{ Moisture, \% } & 0.0 & 2.5 & 7.5 & 15.0 \\
\hline & \multirow{2}{*}{0.40} & 0,24 & 0,22 & 0,20 \\
\hline & & 0,86 & 1,03 & 0,88 \\
\hline
\end{tabular}

Such change is caused by the fact, that a well thermometric conductive soil air is substituted by water, which thermometric conductivity is much less. In humus horizons this process becomes even more complicated. Under the influence of substantial sorbing power the moisture is absorbed into organic and mineral earth fractions, filling their micropores and reducing the number of heat contacts, which causes the increase of conductive thermometric conductivity. As a result, with the growth of moisture it decreases slower than in mineral horizons or in the sand.

The thermometric conductivity, caused by steam transference $\left(a^{*}\right)$ for the investigated samples of chernozem is characterized by extreme values, referring to moisture content close to CBB, and in the quartz sand - to capillary moisture content. This is the degree of soil-moistening which provides the best conditions for thermal and steam conduction, when the concentration of steam molecules is high enough and there is the possibility for their free movement in a porous space.

At the same time, the presence of water cuffs does not make the obstructions for thermotransference, i.e. while dissolving on the "warm" side of the cuff, the steam molecules, moving in an airy pore, condense on the "cold" border of the next water pellicle, accelerating the process of heat transference owing to phase transformations of soil moisture. 
It is necessary to point out that the steam-diffuse constituent in humus horizons of the chernozem plays the less role, than in lower mineral ones, and much less in the quartz sand in comparison with conductive constituent. It shows once again the high mobility of steam molecules in the systems, where the energy of connection of liquid and firm phases is not great. So, in the $B$ horizon of leached chernozem by $C B B$ the thermometric conductivity, caused by steam-transference, is twice larger than the conductive one. In the quartz sand, where the dispersion degree is much less, this exceeding can reach 4 and more times.

Figure 1 presents the interdependence of equivalent (total) and thermometric conductivity, caused by the steam molecules movement, as well as the coefficient of thermal-and-steam conductivity of chernozem and quartz sand depending on the moisture content. The picture shows that the curves of thermal-and-steam conductivity also have extreme values. This proves the existence of the conditions, optimum for thermotransference, arisen by the definite hydrological constant, depending on the dispersion degree.
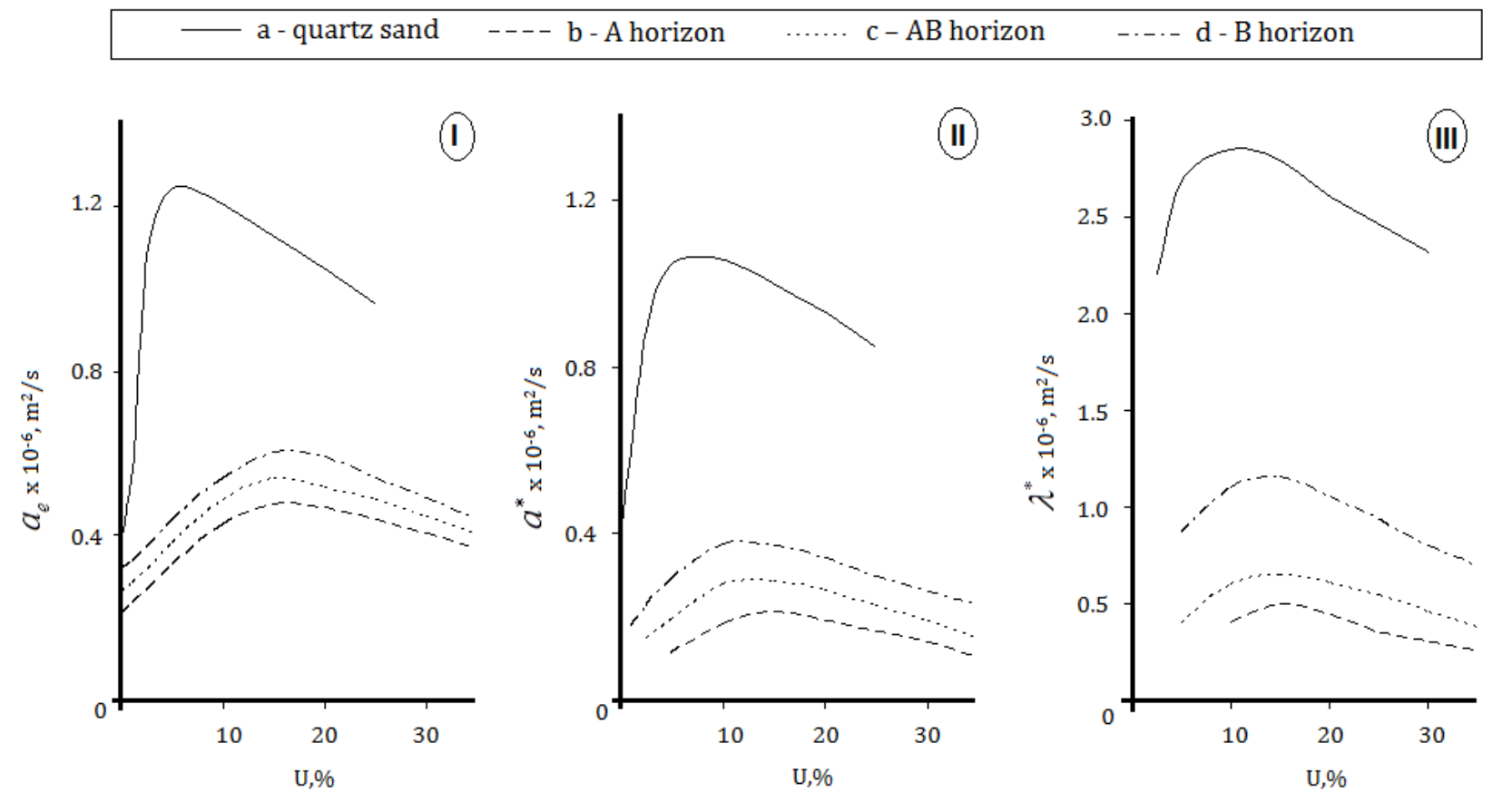

Figure 1. The interdependence of equivalent (total) (I- $a_{e}$ ) and thermometric conductivity (II- $a^{*}$ ), caused by the steam molecules movement, as well as the coefficient of thermal-and-steam conductivity (III- $\lambda^{*}$ ) of chernozem and quartz sand depending on moisture content $(U)$ : $a$ - quartz sand, $b$ - $A$ horizon, $c-A B$ horizon, $d$ - $B$ horizon.

At the same time the coefficient of thermal condition, caused by steam-transference, is many times lower than of the conductive one, which by moistening from absolutely dry condition to $(H B)$ undergoes a change for different horizons of chernozem in the range $(0.3-1.4) \mathrm{W} /(\mathrm{m} \mathrm{K})$

Therefore, the steam molecules, dramatically accelerating the process of thermal conditions in the soil profile, especially in mineral horizons, transfer some heat, promoting a little thermal accumulation in the soil. This was reported by Globus (1983).

\section{Conclusion}

- The solution of system of differential equations lets work out the method of determination of steamdiffuse constituents of the complex mechanism of thermal transference based on the use of impulse method of flat heat source.

- It was shown that the part of thermometric conductivity caused by the steam molecule movement is very substantial in heat-exchange, especially in mineral horizons of chernozem. It also depends very much on the dispersion degree of firm phase of soil. Conductive thermometric conductivity, with the increase of soil moisture content has the tendency to lower because of moisture-substituted soil air.

- The amount of heat transferred by steam is not large, because the thermal-and-steam conduction is many times less than conductive thermal conduction, caused by firm and liquid phases of soil. 


\section{References}

Anikonov, Yu.Ye., Bubnov, B.A., 1981. Existence and uniqueness of solution of inverse problem of parabolic equation. Doklady Akademii Nauk SSSR (The Proceedings of the USSR Academy of Sciences) 289 (4): 777-779 [in Russian].

Arkhangelskaya, T.A., 2014. Diversity of thermal conditions within the paleocryogenic soil complexes of the East European Plain: The discussion of key factors and mathematical modeling. Geoderma 213: 608-616.

Bolotov, A.G., Makarychev, S.V., 2015. Hydrophysical properties of the soils of the south-east of West Siberia. Altai State Agricultural University (ASAU) Publishing Division. Barnaul, Russia. 129 pp. [in Russian].

Chudnovskiy, A.F., 1976. Soil thermal physics. Nauka. Moscow, Russia. 352 pp. [in Russian].

Globus, A.M., 1983. Physics of non-isothermal in-soil moisture exchange. Leningrad: pp. 64-69 [in Russian].

Globus, A.M., 1987. Soil-hydrophysical support of agro-ecological mathematical models. Leningrad. 427 pp. [in Russian].

Gülser, C., Ekberli, I., 2004. A Comparison of estimated and measured diurnal soil temperature through a clay soil depth. Journal of Applied Science 4(3): 418-423.

Kaye, G.W.C., Laby, T.H., 1995. Tables of physical and chemical constants. Longman. 16 th edition, UK. 611 pp.

Lavrentyev, M.M., 1986. Inner problems for differential equations. Partial differential equations. Novosibirsk, Nauka, Russia. pp. 126-129 [in Russian].

Lunin, A.I., 1972. Pulse method of definition thermophysical properties of wet construction materials. Thesis Abstract. Cand. Sci. Moscow. Russia. MISI. 18 pp. [in Russian].

Makarychev, S.V., Mazirov, M.A., 1996. Soil thermal physics: methodology and properties. Vol. 2. VRIA. Suzdal, Russia. 231 pp. [in Russian].

Mikayilov, F.D., Shein, E.V., 2010. Theoretical principles of experimental methods for determining the thermal diffusivity of soils. Eurasian Soil Science 43(5): 556-564.

Nerpin, S.V., Chudnovskiy, A.F., 1970. Physics of the soil (Translated from Russian, 1967) Isreal program for scientific translations, Keter Press, Jerusalem, Israel. pp.194-233.

Panfilov V.P., Makarychev S.V. 1981. Thermophysical properties and regimes of the chernozem soils of the Priobye (Ob River area). Novosibirsk. Nauka. Russia. pp. 18-24 [in Russian].

Philip, J.R., De Vries, D.A., 1957. Simultaneous heat and moisture transfer in porous media. Eos, Transactions American Geophysical Union 38 (2): 222-232.

Tikhonov, A.N., Arsenin, V.Ya., 1979. Methods of solution of incorrect problem. Moskow. Nauka. Russia. 288 pp. [in Russian]. 